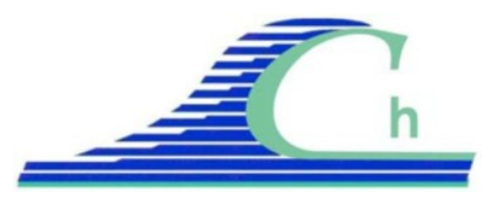

XII ${ }^{\text {ìmes }}$ Journées Nationales Génie Côtier - Génie Civil

Cherbourg, 12-14 juin 2012

DOI:10.5150/jngcgc.2012.115-L @ Editions Paralia CFL

disponible en ligne - http://www.paralia.fr - available online

\title{
Gestion durable de sédiments méditerranéens : influence des propriétés physiques et chimiques sur le comportement mécanique des procédés de $\mathrm{S} / \mathrm{S}$ à base de ciment et de chaux
}

\author{
Yingjie LIANG ${ }^{1}$, Daniel LEVACHER ${ }^{1}$
}

\author{
1. Université de Caen, GRGCE, UMR 6143 CNRS - M2C, 24 rue des Tilleuls, \\ 14000 Caen, France. \\ yingje.liang@unicaen.fr ; daniel.levacher@unicaen.fr
}

\section{Résumé :}

La gestion durable à terre des sédiments marins dragués est une problématique bien actuelle. Ces sédiments ayant le statut de déchets doivent être conditionnés et traités en respect des règles environnementales en vigueur et intégrant la notion de coût économique. La filière de valorisation la plus répandue est celle des matériaux routiers élaborés à base de liants hydrauliques : ciment et chaux, plus ou moins additionnés de cendres volantes. Un travail expérimental de solidification et stabilisation (S/S) de sédiments méditerranéens en sous couches routières a été mené sur la même base de traitement. Les dosages maximaux en liants hydrauliques ont été de $7 \%$ de ciment et $2 \%$ de chaux. Une addition d'un liant pouzzolanique a été proposée avec un ajout de $10 \%$ de cendres volantes comme coproduits industriels. Six sédiments de ports méditerranéens différents ont été étudiés. Ils ont été conditionnés et traités donnant lieu à deux catégories de sédiments : bruts (type I ou B), et dessablés et déshydratés (type II ou DD). Les propriétés physico-chimiques sont données pour les six sédiments; les méthodes de préparation, de conservation et d'essais sont rappelées. Plusieurs formulations ont été testées dans le but d'obtenir des performances suffisantes pour un matériau utilisé en sous couches routières. L’indicateur de résistance retenu est celui donnant la résistance maximale à la rupture en compression simple Rc, pour une valeur seuil de $1 \mathrm{MPa}$. Suivant les formulations, l'analyse de cet indicateur est d'abord donnée et discutée. Puis, selon les différentes caractéristiques physico-chimiques : répartition des grains, limite d’Atterberg, teneurs en matière organique et carbonates des sédiments marins, les diverses influences de ces propriétés sur les performances mécaniques sont rapportées. Ainsi pour les 6 sédiments dessablés et déshydratés, l'évolution des valeurs de résistance Rc diffère selon les teneurs en matière organique ou en carbonates comme il est démontré qu'à court terme, les cendres volantes comme ajout peuvent améliorer ces valeurs de Rc. Les conclusions permettent d'une part, de classer les performances de ces formulations selon l'indicateur de résistance retenu conformément au guide GTR (2000) et d'autre part, de suggérer la construction d'un outil de décision "paramètres déterminants/filières de valorisation" qui serait très utile aux gestionnaires de ports et voies d'eau. 


\section{Mots-clés :}

Sédiments marins - Matière organique - Carbonates - Matériaux routiers - Résistance en compression - Cendres volantes - Solidification/stabilisation

\section{Introduction}

Du point de vue pratique, dans une démarche de gestion durable à terre des matériaux fins que sont les produits de dragage : sables et/ou sédiments qui représentent des volumes considérables à valoriser et des tonnages conséquents, la recherche de filières de valorisation est primordiale. Alors dans un cadre législatif de plus en plus contraignant, toute filière de valorisation doit être organisée, cela passe par un conditionnement et des traitements des produits dragués i.e. stockage, séchage ou déshydratation, décontamination pour aboutir à une valorisation potentielle ou au moins à une élimination vers les centres de stockage de déchets. Dans ces schémas de conditionnement et de traitements, on retrouve les procédés de stabilisation et/ou solidification à base de liants hydrauliques et pouzzolaniques. Et de plus, si la demande en granulats s'accentue, privilégier et développer une filière de valorisation de matériaux fins dragués vers le secteur de la construction apparaît judicieux, ces matériaux élaborés venant se substituer aux granulats terrestres.

C’est dans ce contexte, que plusieurs ports sur la Méditerranée, ont eu à gérer d’importants volumes des sédiments marins dragués plus ou moins contaminés. Les traitements appliqués à ces sédiments l'ont-été pour tenter de les valoriser en matériaux routiers et ce, avec des formulations à base de liants à dosages économiques. S'appuyant sur un référentiel de travaux récents, il apparaît que les mélanges sédiments fins-liants peuvent constituer une voie de valorisation potentielle pour des sous-couches routières et dans la mesure où les teneurs en matières organiques et en métaux lourds dans ces sédiments sont acceptables (COLIN, 2003 ; DUAN, 2008 ; SILITONGA 2010). Puis on peut se référer à quelques conclusions issues de la littérature sur le rôle des liants autres que le ciment. La chaux peut modifier les limites d'Atterberg (LEROUEIL \& LE BIHAN, 1996), la structure et les propriétés d’un sol fin (CAI et al., 2006), elle peut faire augmenter le pH (LIONS, 2004). La chaux peut agir contre la matière organique qui inhibe la réaction d'hydrolyse du ciment (TREMBLAY et al., 2002 ; DUBOIS et al., 2009). Des cendres volantes peuvent être ajoutées à des mélanges à base de chaux pour améliorer les performances mécaniques $(R c)$ à court terme selon CROISE (1964).

Ainsi, diverses formulations de mélanges de sédiments fins méditerranéens avec des liants précédemment cités par divers auteurs : ciment, chaux et cendres volantes ont été réalisées. L'étude de l'influence des caractérisations physico-chimiques des sédiments sur les performances mécaniques obtenues par ce procédé de $\mathrm{S} / \mathrm{S}$ à base des liants a été menée et fait l'objet de cette communication. L’indicateur mécanique pour évaluer l'aptitude à un éventuel emploi de ces mélanges en tant que matériau en sous couches routières est la valeur seuil de résistance $R c$ de $1 \mathrm{MPa}$ en compression simple, établie à 


\section{XII ${ }^{\text {èmes }}$ Journées Nationales Génie Côtier - Génie Civil \\ Cherbourg, 12-14 juin 2012}

une date conventionnelle de 28 jours. Pour tous les mélanges, des éprouvettes cylindriques d'élancement 2, ont été confectionnées pour les 6 sédiments méditerranéens, d'une part, 6 sédiments bruts notés B (type I), et d'autre part, 6 sédiments dessablés et déshydratés notés DD (type II). Des essais de résistance (compression) et de durabilité ont été réalisés aux dates usuelles de 14, 28 et 60 jours. Ici ne sont rapportés et discutés que les résultats des essais de compression.

\section{Matériel et méthodes}

\subsection{Sédiments méditerranéens}

Les 6 échantillons bruts et les 6 échantillons dessablé/déshydratés ont été prélevés dans les ports méditerranéens suivants : Arsenal de Toulon (ARS), Ports de Bandol (BAN), de Cannes (CAN), de Sanary-Lavandou (SAN), de Saint-Mandrier (STM) et le Vieux Port de Marseille (VPM). Le tableau 1 récapitule l'ensemble des principales caractéristiques physico-chimiques des sédiments méditerranéens conditionnés et traités.

Tableau 1.Ppropriétés physico-chimiques des sédiments méditerranéens.

\begin{tabular}{|c|c|c|c|c|c|c|c|c|c|c|c|c|}
\hline \multirow[t]{3}{*}{ Caractérisation } & \multicolumn{12}{|c|}{ Catégorie de sédiments } \\
\hline & ARS & $B A N$ & $C A N$ & SAN & STM & $V P M$ & ARS & $B A N$ & $C A N$ & $S A N$ & STM & $V P M$ \\
\hline & $B$ & $B$ & $B$ & $B$ & B & $B$ & $D D$ & $D D$ & $D D$ & $D D$ & $D D$ & $D D$ \\
\hline \multicolumn{13}{|l|}{ Répartition des grains } \\
\hline Fraction sableuse (>2000 $\mu \mathrm{m}),(\%)$ & 0.7 & 0.2 & 4.4 & 1.0 & 0 & 0 & 0.2 & 1.5 & 0 & 0 & 0 & 0 \\
\hline Fraction sableuse (63 à 2000 $\mu \mathrm{m}$ ), (\%) & 25.4 & 39.6 & 22.6 & 55 & 19.7 & 28.2 & 24.8 & 23.6 & 30.9 & 37.3 & 26.4 & 25.6 \\
\hline Fraction silteuse (2 à 63رm), (\%) & 58.6 & 53.8 & 60.2 & 36.9 & 74.1 & 60.0 & 66.3 & 64.1 & 48.3 & 53.2 & 64.7 & 65.2 \\
\hline Fraction argileuse $(<2 \mu \mathrm{m}),(\%)$ & 15.3 & 6.4 & 12.8 & 7.1 & 6.2 & 11.8 & 8.7 & 10.8 & 20.8 & 9.5 & 8.9 & 9.2 \\
\hline Coefficient d'uniformité $\mathrm{Cu}$ & 19.8 & 19.8 & 26.2 & 38.0 & 9.4 & 19.8 & 13.6 & 21.8 & 60.6 & 26.2 & 16.4 & 15 \\
\hline Coefficient de courbure Cc & 0.7 & 1.0 & 1.1 & 1.3 & 1.0 & 0.9 & 1.0 & 1.3 & 1.0 & 1.3 & 1.2 & 1.1 \\
\hline \multicolumn{13}{|l|}{ Limites d'Atterberg } \\
\hline Limite de liquidité, (\%) & 37 & 31 & 27 & 33 & 121 & 77 & 77 & 71 & 72 & 97 & 118 & 87 \\
\hline Limite de plasticité, (\%) & 30 & 25 & 22 & 32 & 95 & 58 & 59 & 55 & 53 & 58 & 94 & 73 \\
\hline Indice de plasticité, (\%) & 7 & 6 & 5 & 1 & 26 & 19 & 18 & 16 & 19 & 39 & 24 & 14 \\
\hline \multicolumn{13}{|l|}{ Autres constituants } \\
\hline Eau initiale, (\%) & 15 & 18 & 16 & 23 & 70 & 75 & 44 & 42 & 43 & 52 & 89 & 51 \\
\hline Matière organique, (\%) & 17 & 6.5 & 5.7 & 5.7 & 17.2 & 18.2 & 20.5 & 13.1 & 14.8 & 18.5 & 24.1 & 20 \\
\hline Carbonates, (\%) & 32.9 & 27.8 & 16.7 & 23 & 16.5 & 21.2 & 16.8 & 25.9 & 21.6 & 11 & 13.6 & 13.6 \\
\hline
\end{tabular}

\subsection{Liants hydrauliques et pouzolaniques}

Le type de ciment choisi pour les mélanges est un CEM II B-M (S-LL) 32,5R CE CP1 NF. La chaux utilisée est une chaux de marque Dugay, une chaux vive en poudre 0/1.5 mm, de 
teneur en $\mathrm{CaO}$ supérieure à 93\%. Ce sont les liants de base pour les mélanges étudiés, y est ajouté un liant pouzzolanique : une cendre volante. Les cendres volantes retenues sont de la Sodeline ${ }^{\circledR}$ qui est une poudre de couleur gris clair sans odeur. La Sodeline ${ }^{\circledR}$ est une cendre volante qui est de nature silico-alumineuse (voir tableau 2). Elle a une structure très poreuse et se présente sous la forme d'une poudre très fine et légère, assemblage de plaquettes et de fragments alvéolaires.

Tableau 2. Composition chimique de la Sodeline ${ }^{\circledR}$.

\begin{tabular}{lllllllllll}
\hline (\%) & $\mathbf{S i O}_{2}$ & $\mathbf{F e}_{2} \mathbf{O}_{3}$ & $\mathbf{A l}_{2} \mathbf{O}_{3}$ & $\mathbf{M g O}$ & $\mathbf{M n O}_{2}$ & $\mathbf{C a O}_{\text {total }}$ & $\mathbf{C a O}_{\text {libre }}$ & $\mathbf{N a}_{2} \mathbf{O}$ & $\mathbf{K}_{2} \mathbf{O}$ & $\mathbf{S O}_{3}$ \\
\hline Sodeline $^{\circledR}$ & 47.36 & 7.09 & 21.63 & 3.32 & 0.62 & 8.52 & 0.90 & 0.46 & 4.35 & 4.02 \\
\hline
\end{tabular}

\subsection{Préparation, conservation et protocoles}

Le tableau 3 présente les formulations des mélanges retenues pour l'étude de valorisation. Au préalable, les sédiments ont été séchés en étuve à $60^{\circ} \mathrm{C}$. Le séchage ne fait pas partie de l'étude proprement dit, même s’il faudra la considérer du point de vue pratique et économique. Les éprouvettes sont effectuées par compactage manuel dans des moules cylindriques de diamètre $34 \mathrm{~mm}$ (élancement 2), puis conservées à l'air ambiant (salle de laboratoire, $20^{\circ} \mathrm{C} \pm 2^{\circ} \mathrm{C}$. Les performances mécaniques sont déterminées à partir d'essais de résistance à la compression simple $R c j$ à j jours (14, 28 et 60 jours) selon la norme NF EN 12390-3 (2003).

Tableau 3. Composition des mélanges réalisés avec les sédiments méditerranéens.

\begin{tabular}{lllll}
\hline Echantillons & Eau (\%) & Ciment (\%) & Chaux (\%) & Sodeline $^{\circledR}$ \\
\hline Sédiments/B & 30 & 7 & 2 & - \\
Sédiments/B & 30 & 7 & 2 & 10 \\
Sédiments/DD & 50 & 7 & 2 & - \\
Sédiments/DD & 50 & 7 & 2 & 10 \\
\hline
\end{tabular}

\section{Résultats et discussion}

3.1 Analyse de la résistance en compression des mélanges sédiments-ciment-chaux Les premiers mélanges portent sur un traitement de base aux liants hydrauliques : ciment et chaux. L'étude du durcissement dans le temps des mélanges permet d'observer l'évolution aux jeunes âges, à long terme (60 jours) et la stabilité dans le temps, des résistances. Tout d'abord la figure 1 montre cette évolution pour tous les sédiments bruts (B) et dessablés/déshydratés (DD) stabilisés avec $7 \%$ de ciment et $2 \%$ de chaux, en fonction du temps pour des essais réalisés à 14, 28 et 60 jours. Il s'agit de valeurs moyennes (3 essais) à la rupture. Les valeurs observées de $R c$ des sédiments bruts et dessablés/déshydratés ARS, BAN, CAN et SAN sont les plus élevées et supérieures à 


\section{XII ${ }^{\text {èmes }}$ Journées Nationales Génie Côtier - Génie Civil \\ Cherbourg, 12-14 juin 2012}

1 MPa à 14, 28 et 60 jours. Pour les deux autres sédiments bruts et dessablés/déshydratés STM et VPM, les valeurs de $R c$ apparaissent comme les plus faibles et en deçà du seuil requis de $1 \mathrm{MPa}$. Même si on note dans le cas des sédiments STM/DD des valeurs de $R c$ de 1.38 MPa et 1.29 MPa respectivement à 14 et 28 jours; la valeur à 60 jours n'est plus que de $0.98 \mathrm{MPa}$. Disons que le comportement mécanique de ces deux sédiments VPM et STM diffère des 4 autres. Une autre remarque concerne le conditionnement et le traitement avant la stabilisation (mélanges). En effet, les valeurs des $R c$ obtenues pour les sédiments bruts sont plus stables dans le temps que celles obtenues les sédiments dessablés/déshydratés après 28 jours. Ceci pose la question d'effectuer ou non une phase de déshydratation et dessablage avant la stabilisation aux liants. Il s'avère que la granulométrie des deux catégories $\mathrm{B}$ ou DD est restée très proche, $0 / 2 \mathrm{~mm}$.
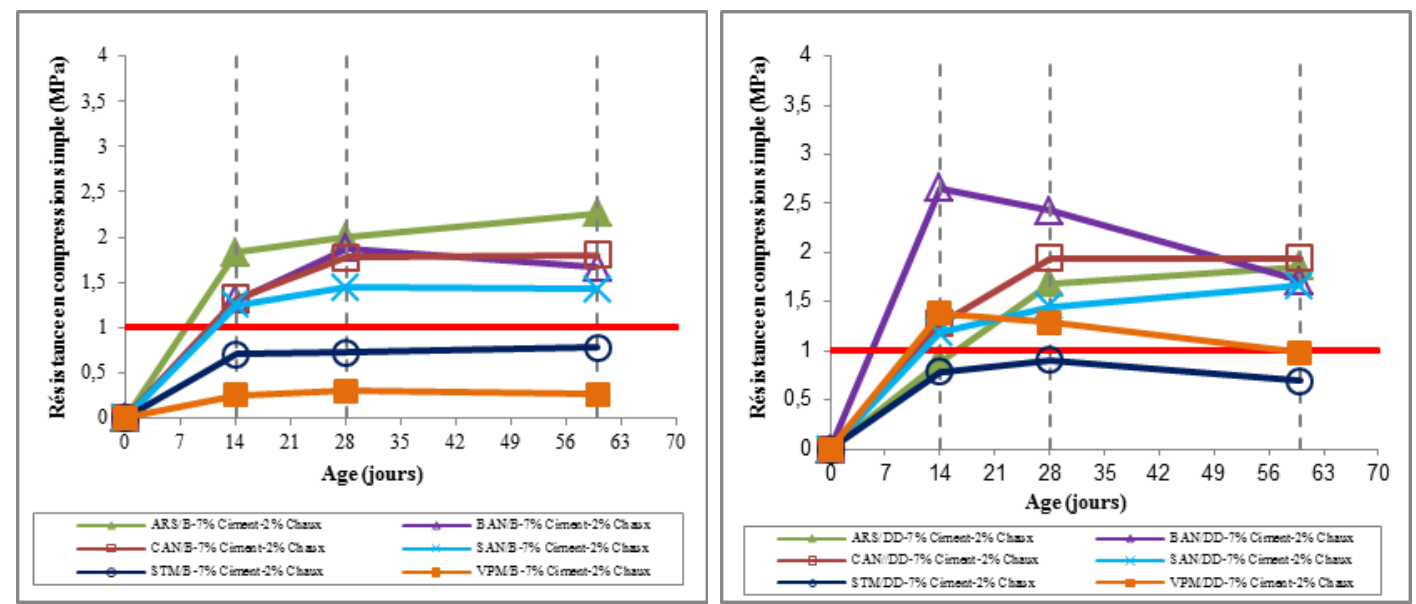

Figure 1. Evolution comparée des résistances à la compression pour les formulations 7\% ciment-2\% chaux des sédiments méditerranéens.

3.2 Analyse de la résistance en compression des mélanges sédiments-ciment-chauxcendres volantes

Si l'on observe la figure 2, l'évolution des résistances, on note une nette amélioration de l'ensemble des valeurs de Rc. Ceci est consécutif à l'ajout de cendres volantes (CV). En effet, pour des dosages correspondant à $7 \%$ de ciment, $2 \%$ de chaux et $10 \%$ de cendres volantes, et comparativement à la figure 1 , les valeurs obtenues de $R c$ pour ces mélanges, sont plus élevées. Cependant, après 28 jours, les valeurs des $R c$ tendent à diminuer plus vite, voir figure 2. Des différences dans le comportement sont à noter, comme pour les valeurs de $R c$ des sédiments bruts ARS/B et VPM/B qui, avec un ajout de $10 \%$ de CV augmentent beaucoup. Dans le cas des sédiments dessablés/déshydratés, ce sont les sédiments CAN/DD et VPM/DD qui, avec 10\% de CV, fournissent des valeurs moyennes plus élevées que celles obtenues sans CV. 
Si l'on considère les teneurs en argiles et en matière organique dans le cas des sédiments ARS/B, VPM/B, CAN/DD et STM/DD, on observe que les cendres volantes ajoutées peuvent contribuer à l'amélioration des résistances $R c$ des sédiments dont la teneur en argiles et en matière organique est élevée.
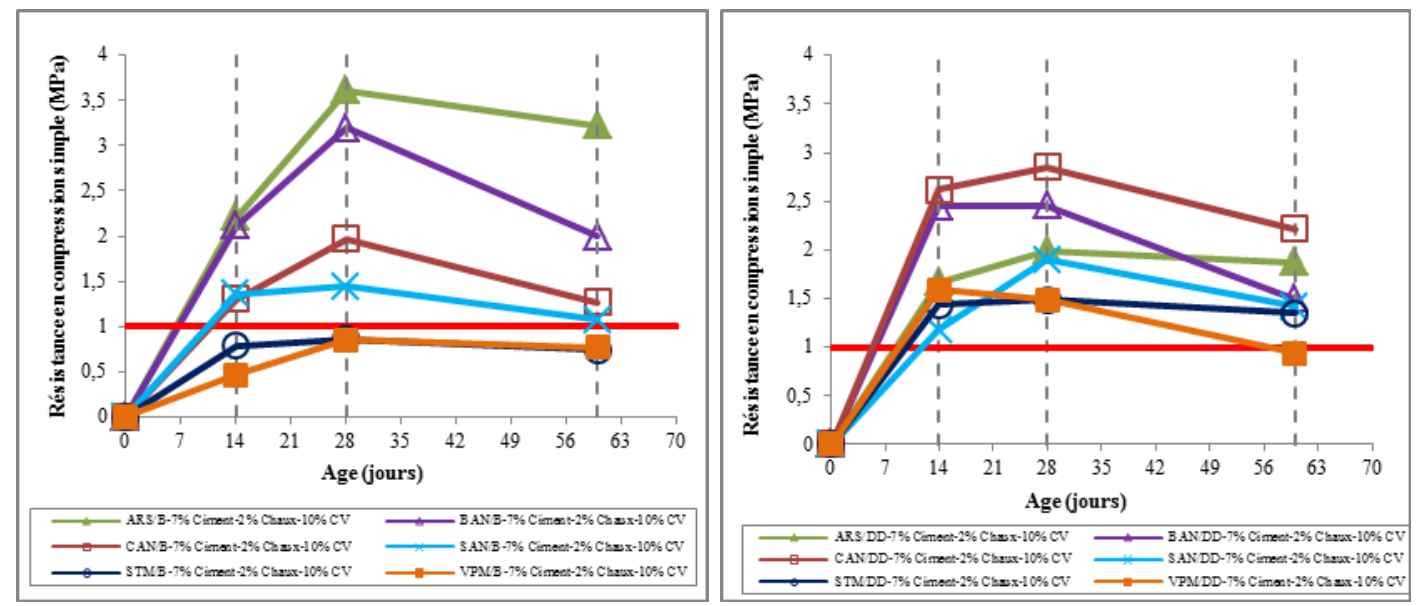

Figure 2. Evolution comparée des résistances à la compression pour les formulations 7\% ciment-2\% chaux-10\% cendres volantes des sédiments méditerranéens.

\subsection{Effet de la teneur en matière organique sur la stabilisation des sédiments par liants}

Une influence des matières organiques est constatée. Elle rejoint en cela les conclusions de certains auteurs, (TREMBLAY et al., 2002). Ce paramètre "teneur en matière organique -MO-" est un paramètre déterminant dans l'orientation de filière de valorisation, et notamment pour celle des matériaux routiers. Il y a si l'on veut organiser ces orientations, un seuil ou une teneur en MO limite à définir.

Tout d'abord en étudiant ces mélanges, et selon la matière organique contenue dans les sédiments méditerranéens, on a pu observer que les valeurs des $R c$ à 28 jours se révèlent être les plus faibles. Cet effet est très marqué, et de manière continue, avec les sédiments déshydratés/dessablés quel que soit le mélange en liant: ciment-chaux ou ciment-chaux-CV, voir figures 3 et 4 . Il y a une décroissance de $R c$ au fur et à mesure que la teneur en matière organique augmente. Dans le cas des sédiments bruts, cette tendance est moins observable, ceci peut être à rapprocher à la variabilité de la répartition de la matière organique sur les sédiments bruts, voir figures 3 et 4 . Par ailleurs, on note que pour un taux en matière organique de l'ordre de 15 à $20 \%$ pour les mélanges ciment-chaux, le seuil de $1 \mathrm{MPa}$ serait difficile à atteindre. 

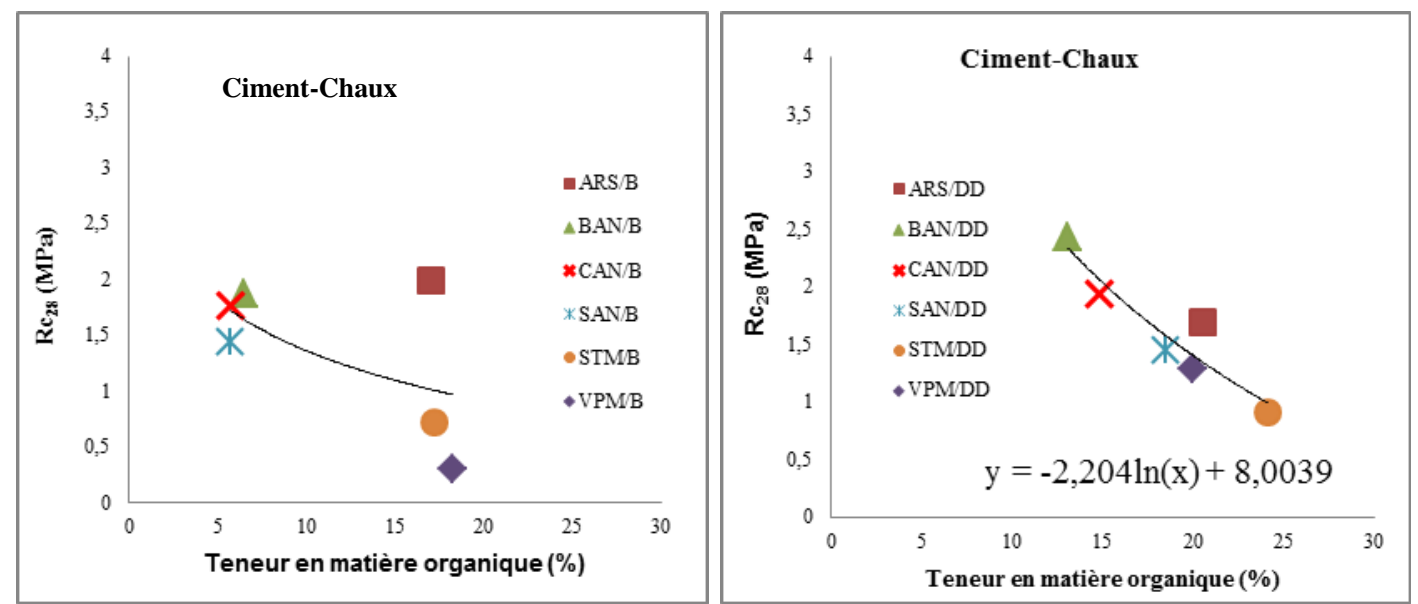

Figure 3. Relation Rc28-matières organiques pour les formulations $7 \%$ ciment-2\% chaux des sédiments méditerranéens.
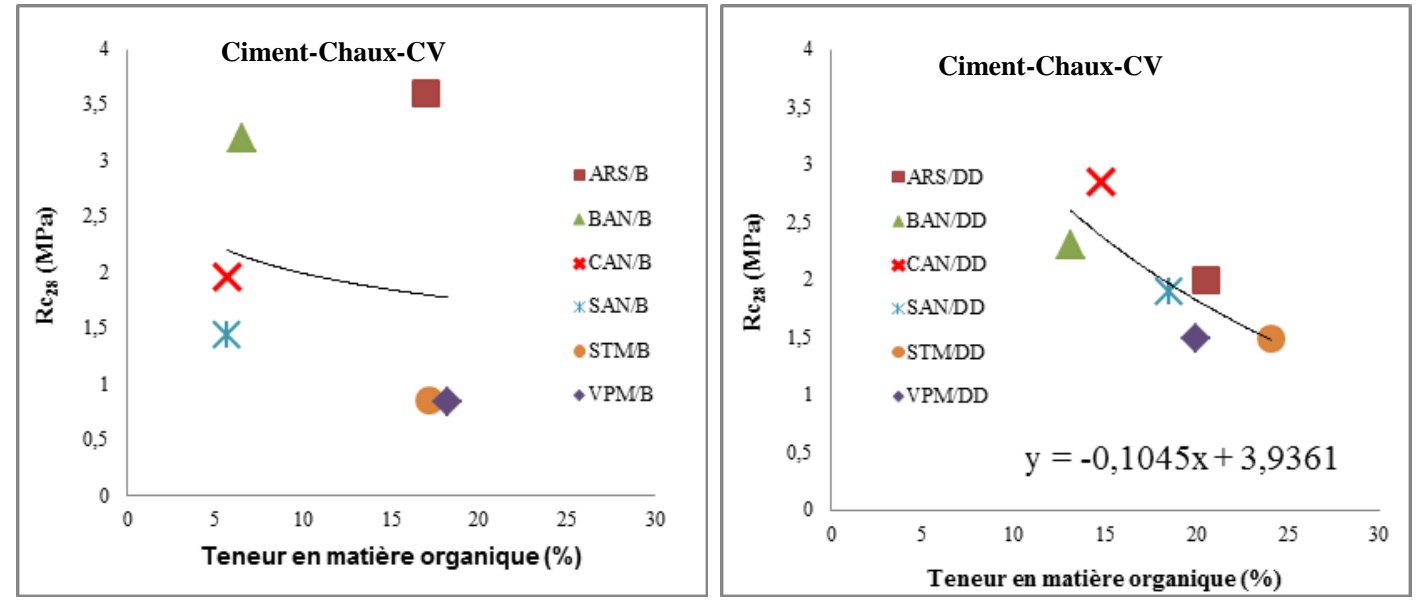

Figure 4. Relation de Rc28-matières organiques pour les formulations 7\% ciment-2\% chaux-10\% cendres volantes des sédiments méditerranéens.

\subsection{Effet de la teneur en carbonates sur la stabilisation des sédiments par liants}

De même si l'on s'intéresse à la teneur en carbonates dans le cas d'une stabilisation par liants, on observe une relation entre les résistances et cette teneur. La teneur en carbonates influe bien sur les valeurs des résistances $R c$ des sédiments méditerranéens quels que soient les mélanges ciment-chaux ou ciment-chaux-CV, voir sur les figures 5 et 6 . Il y une nette tendance croissante des valeurs de $R c$ avec la teneur en carbonates pour les sédiments stabilisés aux liants. 

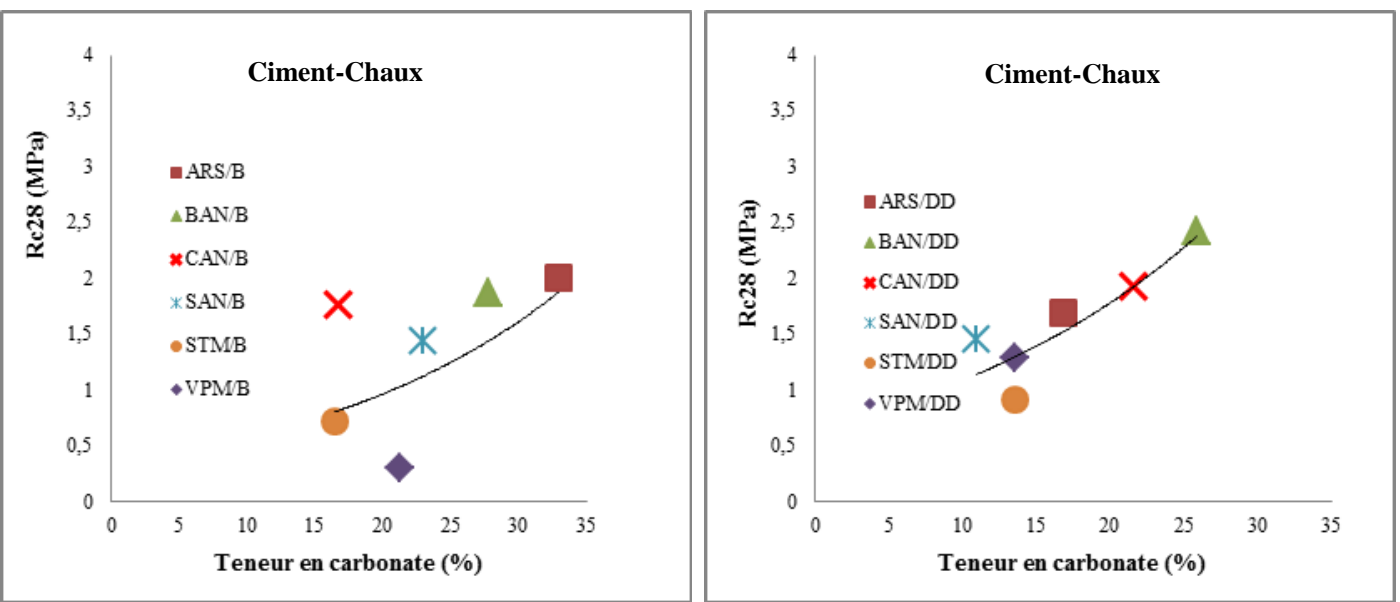

Figure 5. Relation de Rc28-carbonates pour les formulations

$7 \%$ ciment-2\% chaux des sédiments méditerranéens.
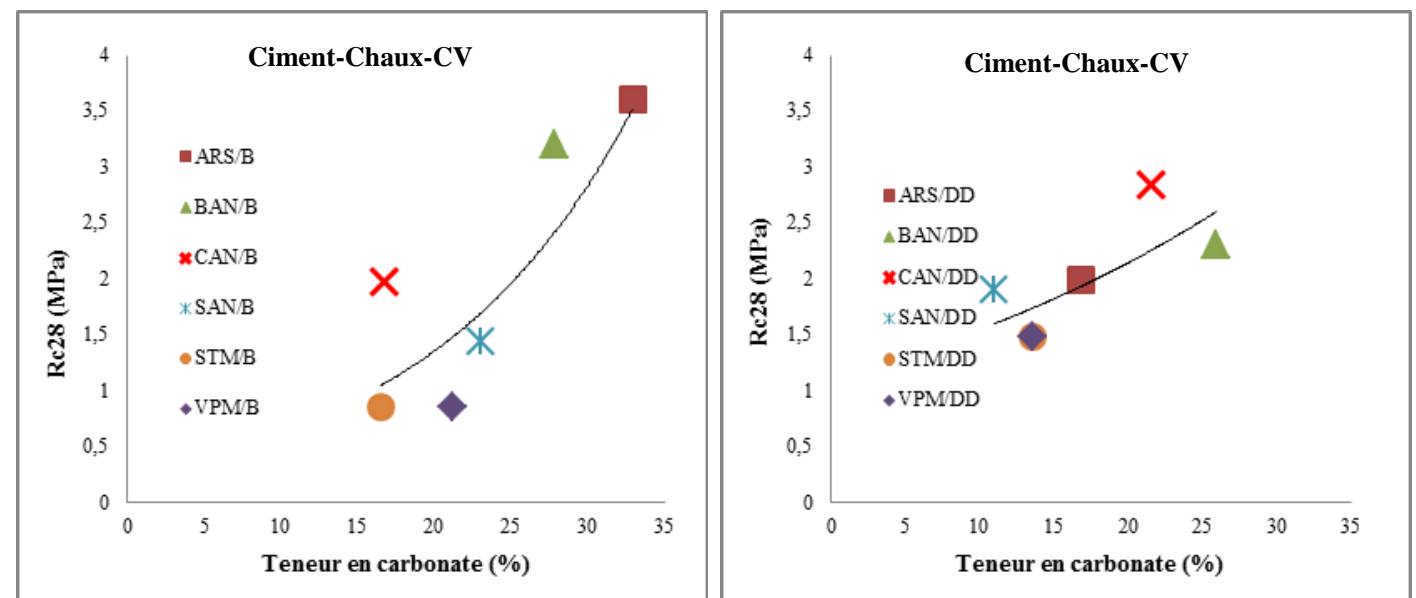

Figure 6. Relation de Rc28-carbonates pour les formulations 7\% ciment-2\% chaux-10\% CV des sédiments méditerranéens.

On peut ajouter que la granulométrie joue aussi un rôle. Si l'on se réfère au tableau 1 , les sédiments bruts ARS, BAN, CAN et SAN, dont les grains sont plus grossiers, possèdent quelques particules de taille supérieure à $2 \mathrm{~mm}$ malgré les faibles quantités comme les sédiments bruts CAN (4.4\%, >2 mm). Pour ces sédiments bruts, les teneurs en matière organique et carbonates influent moins sur les valeurs de $R c$. Ceci peut être le cas des sédiments bruts ARS dont la teneur en MO est la plus élevée et les valeurs $R c$, les plus fortes.

\section{Conclusions}

La teneur en matière organique en quantité importante influe sur le comportement mécanique de sédiments marins stabilisés par liants. Un ajout de cendres volantes à un traitement à base de ciment et de chaux peut améliorer les performances mécaniques 


\section{XII ${ }^{\text {èmes }}$ Journées Nationales Génie Côtier - Génie Civil \\ Cherbourg, 12-14 juin 2012}

surtout pour les sédiments à teneur élevée en argiles en matière organique à court terme ( $<28$ jours). Mais dans ce cas, on observe aussi, un fléchissement des valeurs de $R c$ au-delà de 28 jours.

La teneur en carbonates joue un rôle sur l'augmentation du $\mathrm{pH}$, suivant la relation :

$\mathrm{CaCO}_{3}+\mathrm{CO}_{2}+\mathrm{H}_{2} \mathrm{O}=\mathrm{Ca}^{2+}+2\left(\mathrm{HCO}^{3-}\right)$

Le taux de carbonates exprimé en $\mathrm{CaCO}_{3}$, après réaction chimique, donne des ions hydrogénocarbonates $\mathrm{HCO}^{3^{-}}$qui sont faiblement alcalins. La teneur en carbonates dans les sédiments peut aussi diminuer la mobilité des ions des métaux lourds, être favorable à la neutralité et à la diminution des composants organiques dans l'accumulation des matières organiques (VALLS \& VAZQUEZ, 2000 ; LIONS, 2004).

\section{Références}

CAI Y., SHI B., CHARLES W.W., TANG C. (2006). Effect of polypropylene fibre and lime admixture on engineering properties of clayey soil. Engineering Geology, Vol. 87, pp 230-240. doi:10.1016/j.enggeo.2006.07.007

COLIN D. (2003). Valorisation de sédiments fins de dragage en technique routière. Thèse de doctorat, Université de Caen, 2003, 181 p.

DUAN Z. (2008). Caractérisation, stabilisation et solidification de sédiments fins marins. Thèse de doctorat, Université de Caen, 2008, 145 p.

GTR (2000). Guide des Terrassements Routiers, réalisation de remblais et des couches

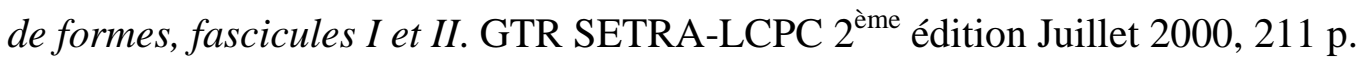

CROISE R. (1964). Traitement des sols aux liants hydrauliques. Ecole Nationale du Génie Rural, des Eaux et des Forêts, Nancy, pp 427-438.

DUBOIS V., ABRIAK N.E., ZENTAR R., BALLIVY G. (2009). The use of marine sediments as a pavement base material. Waste Management, Vol. 29, pp 774-782. doi:10.1016/j.wasman.2008.05.004

LEROUEIL S., LE BIHAN J.-P. (1996). Liquid limits and falling cones. Canadian Geotechnical Journal, 33, pp 793-798. doi:10.1139/t96-104-324

LIONS J. (2004). Etude hydrogéochimique de la mobilité de polluants inorganiques dans des sédiments de curage mis en dépôt : expérimentations, études in situ et modélisation. Thèse de doctorat, École Nationale Supérieure des Mines de Paris. France, 248 p. NF EN 12390-3 (2003). Essai pour béton durci. Partie 3 : résistance à la compression des éprouvettes (indice de classement : P18-455). Février 2003.

SILITONGA E. (2010). Valorisation des sédiments marins contaminés par solidification/stabilisation à base de liants hydrauliques et de fumée de silice. Thèse de Doctorat, Université de Caen, 227 p.

TREMBLAY H., LEROUEIL S., LOCAT J. (2002). Influence of the nature of organic matter on the soil stabilization with cement. Canadian Geotechnical Journal, Vol.39: pp 535-546. doi:10.1139/02-002 
Thème 8 - Gestion et valorisation des sédiments marins

VALLS S., VAZQUEZ E. (2000). Stabilization and solidification of sewage sludge with Portland cement. Cement and Concrete Research, Vol. 30, pp 1671-1678. doi:10.1016/S0008-8846(00)00363-X 